\title{
Thermal Undulations of Lipid Bilayers Relax by Intermonolayer Friction at Submicrometer Length Scales
}

\author{
S. A. Shkulipa, W. K. den Otter,* and W. J. Briels \\ Computational Biophysics, Faculty of Science and Technology, University of Twente, \\ P.O. Box 217, 7500 AE Enschede, The Netherlands \\ (Received 23 December 2005; published 3 May 2006)
}

\begin{abstract}
The time correlation functions of the thermal undulations of a lipid membrane have been studied by molecular dynamics simulations of a coarse-grained bilayer model. We observe a double-exponential decay, with relaxation rates in good agreement with the theory by Seifert and Langer, [Europhys. Lett. 23, 71 (1993)]. Intermonolayer friction resulting from local velocity differences between the two monolayers is shown to be the dominant dissipative mechanism for fluctuations with wave lengths below $\sim 0.1 \mu \mathrm{m}$.
\end{abstract}

DOI: 10.1103/PhysRevLett.96.178302

PACS numbers: 61.20.Ja, 82.70.Uv, 87.16.-b

Biological membranes are amphiphilic bilayers composed of lipids interspersed with fatty acids, sterols, and proteins. They serve to isolate the contents of the cell from the outside world and to separate compartments within the cell. Besides transmitting chemical stimuli by means of their proteins [1], they offer the possibility of transporting material from one side to the other by simple diffusion [2], through channels [2], or by budding and subsequent pinching off of the bud [3]. Obviously they provide mechanical support to their contents. The mechanical and thermodynamic properties of membranes have therefore been studied intensively through the past few decades [4-7]. From a theoretical point of view it is attractive to attribute these properties to a mathematical surface, the neutral surface [8], as, for example, in the celebrated Helfrich free energy [9]

$$
F=\int\left[2 \kappa H^{2}+\bar{\kappa} K+(k / 2)\left(\phi / \phi_{0}-1\right)^{2}\right] d a .
$$

The integral is over the surface of the membrane, $H$ is the local mean curvature, $K$ the local Gaussian curvature, and $\phi$ the local density. $\kappa$ and $\bar{\kappa}$ are the bending rigidity and saddle-splay modulus, respectively, $k$ is the compressibility modulus, and $\phi_{0}$ is the equilibrium density. In the sequel the Gaussian curvature plays no role and will be omitted.

In combination with the relevant hydrodynamic equations, the Helfrich free energy may be used to study the dynamics of membranes at large length scales [10-16]. In many cases of interest, however, small scale deformations of the membrane occur, which force the two monolayers to slip along one another [17] with velocity difference $\Delta v$, giving rise to an intermonolayer friction force per unit area $F_{\text {slip }}=b \Delta v$, with friction coefficient $b$. This happens, for example, in erythrocytes as they wriggle through narrow passageways [4], during the pulling of tethers by kinesine motor proteins [18], in cleavage of cells during the last stage of cell division [2], and in the formation of vesicles from existing membranes by budding [3]. Several experiments have been performed to measure the friction coefficient $b$. Evans and Yeung [19] and Raphael and Waugh [20] reported on tether pulling experiments, while Chizmadzhev et al. [21] induced slippage by pulling a bilayer through a toroidal fusion pore. Typical values of the friction coefficients obtained this way are $b=$ $10^{8}-10^{9} \mathrm{~N} \mathrm{~s} / \mathrm{m}^{3}$ for a range of lipids bilayers.

The theory describing the dynamics of an undulating bilayer with slipping monolayers was first discussed by Seifert and Langer [22,23]. Independently, 1 year later, Evans and Yeung $[19,24]$ published a similar theory for undulating vesicles, which has since been extended $[25,26]$. To the best of our knowledge, only two papers so far on measurements of shape fluctuations have provided some, still inconclusive, support for these theories $[27,28]$. Previous simulations of the dynamics of bilayer fluctuations have not been analyzed with the appropriate equations [29].

By ignoring peristaltic modes, i.e., by fixating the distance between the neutral surfaces of the two monolayers to a value of $2 d$, the degrees of freedom of the bilayer reduce to three fields. One is the position $h(x, y)$ of the midsurface, i.e., the surface halfway between the two monolayers. The other two fields are the two monolayer densities $\phi^{ \pm}(x, y)$. It is convenient to use densities which are projected on the midsurface [6,22,23], $\psi^{ \pm}=\phi^{ \pm}(1 \mp$ $2 d H$ ), since it is the difference of the velocities corresponding to these densities which determines the intermonolayer friction. This can be understood by noticing that a uniform bending of the bilayer should not result in any friction. Finally, we introduce the normalized density difference $\rho^{\Delta}(x, y)=\left(\psi^{+}-\psi^{-}\right) / \phi_{0}$ and average density $\rho^{\Sigma}(x, y)=\left(\psi^{+}+\psi^{-}\right) / \phi_{0}-1$, and notice that fluctuations of the latter are decoupled from those of the other degrees of freedom [22]. This leaves us with the coupled dynamics of two fields, $h(x, y)$ and $\rho^{\Delta}(x, y)$. After a Fourier transformation of the appropriate force balances, Seifert 
and Langer arrived at $[6,22,23]$

$$
\frac{\partial}{\partial t}\left(\begin{array}{c}
h_{\mathbf{q}} \\
\rho_{\mathbf{q}}^{\Delta}
\end{array}\right)=\left(\begin{array}{cc}
1 / 4 \eta q & 0 \\
0 & q^{2} / 4 b
\end{array}\right)\left(\begin{array}{cc}
\tilde{\kappa} q^{4} & -k q^{2} d \\
-k q^{2} d & k
\end{array}\right)\left(\begin{array}{l}
h_{\mathbf{q}} \\
\rho_{\mathbf{q}}^{\Delta}
\end{array}\right),
$$

where $t$ is the time, $\tilde{\kappa}=\kappa+d^{2} k$, and $\eta$ is the viscosity of the solvent surrounding the bilayer. The vectors represent the conformation of the bilayer in Fourier space, with $\mathbf{q}$ being the wave vector of the Fourier components and $q$ its length. Modes with different wave vectors are decoupled. The second matrix on the right-hand side is the Hessian of the free energy of the bilayer expressed in the complex Fourier components $h_{\mathbf{q}}$ and $\rho_{\mathbf{q}}^{\Delta}$ of the fields $h(x, y)$ and $\rho^{\Delta}(x, y)$. The first matrix is called the mobility matrix. The final solution follows after diagonalization of the product of the two matrices.

We now invoke Onsager's regression hypothesis [30], in combination with the above theory, to describe the dynamics of the fluctuations of a bilayer around its equilibrium state. The height-height time correlation function then reads [6]

$$
\left\langle h_{\mathbf{q}}(t) h_{\mathbf{q}}^{*}(0)\right\rangle=A_{1}^{h} e^{-\gamma_{1} t}+A_{2}^{h} e^{-\gamma_{2} t},
$$

where the angular brackets denote a canonical average and the asterisk indicates a complex conjugate. $A_{1}^{h}$ and $A_{2}^{h}$ are two $q$-dependent amplitudes [6]. The relaxation rates $\gamma_{1}$ and $\gamma_{2}$ are the smallest and the largest eigenvalue, respectively, of the product of the two matrices in Eq. (2). Their dependence on the wave number $q$ is shown in Fig. 1, using particular values for the various parameters pertaining to our coarse-grained model, to be described below. The curves exhibit an avoided crossing around a critical wave number $q_{c}=\eta k / b \tilde{\kappa}$, similar to those found in phonon dispersion curves. Inspection of the corresponding eigenvectors $[6,22]$ reveals that the slow mode has bending character at low wave numbers and slipping character at large wave numbers, as indicated in the figure. The opposite holds true for the fast mode. The bending part of the slow mode behaves like $\gamma_{1}=\kappa q^{3} / 4 \eta$ and that of the fast mode like $\gamma_{2}=\tilde{\kappa} q^{3} / 4 \eta$. For the slipping branches one finds $\gamma_{2}=k q^{2} / 4 b$ at small wave numbers and $\gamma_{1}=$ $k \kappa q^{2} / 4 b \tilde{\kappa}$ at high wave numbers. In the limit of zero thickness, $d=0$, the avoided crossing disappears and the two bending parts merge into one line with $\gamma=\kappa q^{3} / 4 \eta$, also described by the older theories [10-13], while the slipping parts merge into one line with $\gamma=k q^{2} / 4 b$, not treated in the older theories.

Simulations $[31,32]$ were done using a simple coarsegrained model [33], in which the amphiphiles are represented by short chains of one head particle and four tail particles, connected by harmonic springs and a bending potential. The solvent consists of loose particles. Nonbonded interactions between particles are of the LennardJones type, $\phi_{\mathrm{LJ}}=4 \epsilon\left[(r / \sigma)^{-12}-(r / \sigma)^{-6}\right]$, except for a purely repulsive potential $\phi_{\text {rep }}=\epsilon[r /(1.05 \sigma)]^{-9}$ model-

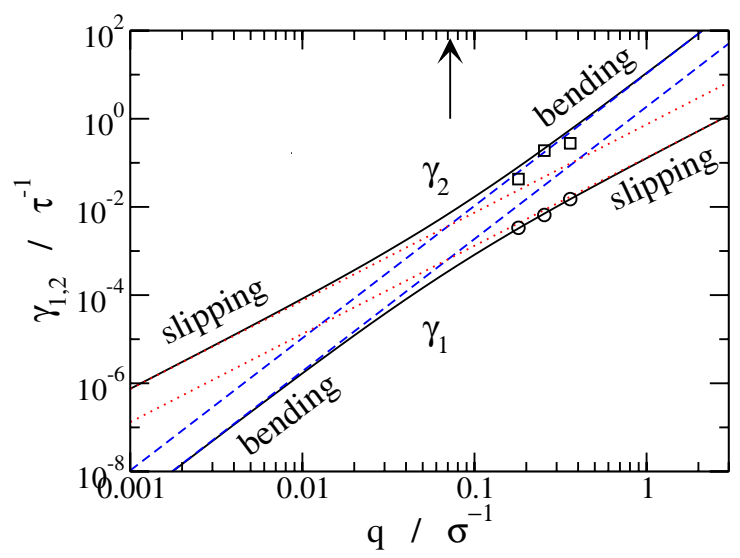

FIG. 1 (color online). The slow $\gamma_{1}$ and fast $\gamma_{2}$ relaxation rates (solid lines) of an undulating bilayer as functions of the wave number $q$, calculated using the parameters of the coarsegrained model. The slipping branches scale as $q^{2}$ and are damped by intermonolayer friction, the bending branches scale as $q^{3}$ and relax through viscous dissipation by the solvent. Dashed and dotted lines denote extrapolations of the limiting regimes at low and high $q$; the vertical distance between each set of parallel lines is $\log (\tilde{\kappa} / \kappa)$. The arrow indicates the crossover wave number $q_{c}$, where the relaxation characters of the slow and fast modes are gradually exchanged. Circles and squares mark the relaxation rates obtained by fitting the simulated height correlation functions of Fig. 2.

ing the hydrophobic interactions. A Nosé-Hoover thermostat was used to maintain a temperature of $1.35 \epsilon / k_{B}$. Indicative translations of simulation results to experimental values are obtained by using [33] $\epsilon=2 \mathrm{~kJ} / \mathrm{mol}, \sigma=$ $1 / 3 \mathrm{~nm}$, and the particle mass $m=36$ a.u., which imply a unit of time $\tau=1.4 \mathrm{ps}$. This particular model is one of the best-studied coarse-grained models available, with previous simulations focusing on its bending rigidity [3436], apparent [33] and intrinsic [36] compressibility, edge energy [37], and the free-energy profile of pore formation [38]. It is the first model for which the surface viscosity and the intermonolayer friction have been established, using nonequilibrium simulations [39]. As expected for a simple coarse-grained model with a single short tail, its dynamical properties turned out to be some 2 orders of magnitude faster than the experimental data on amphiphiles with two longer tails. In combination with its inherent simplicity, this makes the model particularly appealing for exploratory studies like the current study on the importance of intermonolayer friction on the thermal undulations of a bilayer. Figure 1 was calculated using $k=11 \epsilon \sigma^{-2}, \kappa=7.5 \epsilon, b=$ $3.7 \epsilon^{1 / 2} m^{1 / 2} \sigma^{-3}, \eta=1.0 \epsilon^{1 / 2} m^{1 / 2} \sigma^{-2}$, and an estimated $d=t / 4$ for a bilayer of thickness $t=7 \sigma$.

The simulated system comprised a 1152 amphiphile bilayer and 10800 solvent particles at a number density of $2 / 3 \sigma^{3}$ in a periodic simulation box. The area of the square $x y$ ground plane of the box, oriented parallel to the bilayer, was adjusted until the average pressures parallel 
and perpendicular to the bilayer were equal, establishing a tensionless state.

In Fig. 2 we present the calculated time dependent height autocorrelation functions for the three longest wavelengths commensurable with the dimensions of the simulation box. For each of these wave numbers, $q_{1}=0.18 \sigma^{-1}, q_{2}=0.25 \sigma^{-1}$, and $q_{3}=0.36 \sigma^{-1}$, there are two allowed wave vectors, which were both used to produce the averaged curves shown in the figure. The dashed lines are the best representations of the data using two exponentially decaying terms. As usual, because of time reversal symmetry, the early parts of the curves do not follow the macroscopic theory [30] and must be deleted from the fitting procedure. At the lowest wave number two regimes can clearly be discerned, while with increasing wave number the first regime becomes too short to be measured accurately. The second, slow regime in all cases extends over large enough time intervals to allow a precise measurement of the corresponding characteristic times $\gamma_{1}^{-1}$. The results are represented in Fig. 1 by circles, and agree perfectly well with the rates predicted by the theory of Seifert and Langer. Also shown in Fig. 1 are the calculated fast relaxation rates $\gamma_{2}$. Given the difficulties with determining their values, they agree fairly well with the theoretical predictions. These results provide strong evidence for the validity of the theory by Seifert and Langer $[22,23]$.

As is clear from Fig. 1, the slowest relaxations at the wave numbers studied in this Letter have mainly slipping character. The main shortcoming of the model used in our simulations is a value of $b$ which is smaller than typical experimental values by a factor of about 100 . A drawing of the dispersion curves with this larger value of $b$, and

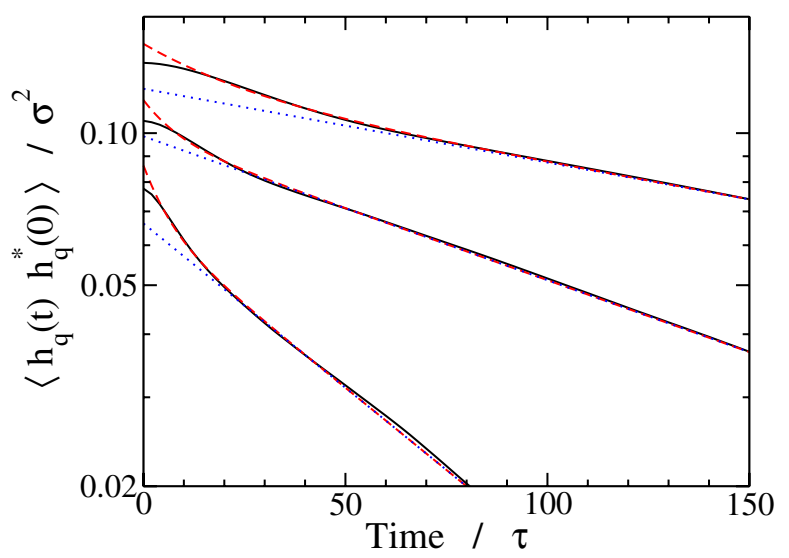

FIG. 2 (color online). Time correlation functions of the Fourier components of the height of a thermally fluctuating bilayer (solid lines), for the three smallest wave numbers commensurable with the box dimension, $q_{1}=0.18 \sigma^{-1}$ (top), $q_{2}=0.25 \sigma^{-1}$ (middle, multiplied by 2.5), and $q_{3}=0.36 \sigma^{-1}$ (bottom, multiplied by 8 ). The dashed lines are double-exponential fits, excluding the transient part of the simulation data; the dotted straight lines show the contributions of the slow modes to these fits. otherwise the same parameters as used before, is depicted in Fig. 3. The bending parts of the plot remain unchanged, while the slipping parts move downwards along the vertical axis since in the latter regimes $\gamma_{1,2} \propto b^{-1}$. As a result, the critical wave number moves to lower values as well. Using realistic values for all membrane parameters we obtain $q_{c} \approx 0.01 \mathrm{~nm}^{-1}$, indicating that the relaxation of membrane fluctuations at length scales below $\sim 0.1 \mu \mathrm{m}$ is dominated by intermonolayer friction. Note that many experiments $[14,40,41]$ probe wave numbers at larger length scales, and therefore sample undulations which mainly relax through energy dissipation by the viscous solvent.

We draw one more conclusion from the above discussion, concerning particle based simulations of bilayers. In simulations of systems with realistic values for the intermonolayer friction coefficient, the longest relaxation time will often be too long to be sampled sufficiently well, even if one chooses small systems to exploit their relatively small relaxation times. Since the usual way to measure the bending rigidity $[29,34,35,42]$ assumes that all fluctuations are fully sampled, such calculations, when applied to atomistic simulations, must be met with care.

The main results of this Letter are a confirmation of the theory by Seifert and Langer describing the relaxation dynamics of undulations in lipid bilayers, and the conclusion, illustrated by the presented molecular dynamics simulations, that at length scales below $\sim 0.1 \mu \mathrm{m}$ the relaxations are dominated by intermonolayer friction.

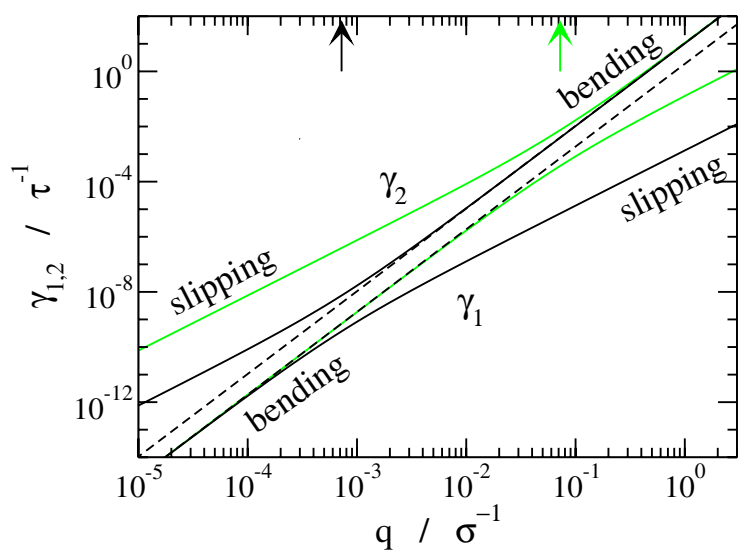

FIG. 3 (color online). The relaxation rates of the coarsegrained model before (gray) and after (black) a 100-fold increase of the intermonolayer friction, to bring this parameter on par with experimental values. Changing $b$ does not affect the relaxation rates of the bending branches, whose limits are indicated by dashed lines. The rates of the slipping branches, however, are reduced by a factor of 100 , and the crossover wave number (arrows) is reduced accordingly. Atomistic and coarsegrained simulations live in the top-right corner of the plot, as these systems typically contain $10^{3}$ lipids, corresponding to $q \sim$ $0.1 \sigma^{-1}$, with simulation times usually of the order of $10^{4} \tau$. 
This work is part of the SoftLink research program of the Stichting voor Fundamenteel Onderzoek der Materie (FOM), which is financially supported by the Nederlandse Organisatie voor Wetenschappelijk Onderzoek (NWO).

*Electronic address: w.k.denotter@utwente.nl

[1] L. Stryer, Biochemistry (W. H. Freeman, New York, 1995), 4th ed.

[2] W. M. Becker and D. W. Deamer, The World of the Cell (Benjamin/Cummings, Redwood City, CA, 1991).

[3] J. S. Bonifacino and B. S. Glick, Cell 116, 153 (2004).

[4] E. A. Evans and R. Skalak, Mechanics and Thermodynamics of Biomembranes (CRC, Boca Raton, FL, 1980).

[5] W. M. Gelbart, A. Ben-Shaul, and D. Roux, Micelles, Membranes, Microemulsions, and Monolayers (SpringerVerlag, New York, 1994).

[6] U. Seifert, Adv. Phys. 46, 13 (1997).

[7] D. Boal, Mechanics of the Cell (Cambridge University Press, Cambridge, England, 2002).

[8] S. A. Safran, Statistical Mechanics of Surfaces, Interfaces and Membranes (Addison-Wesley, Reading, MA, 1994).

[9] W. Helfrich, Z. Naturforsch. C 28, 693 (1973).

[10] L. Kramer, J. Chem. Phys. 55, 2097 (1971).

[11] F. Brochard and J.F. Lennon, J. Phys. (Paris) 36, 1035 (1975).

[12] S. T. Milner and S. A. Safran, Phys. Rev. A 36, 4371 (1987).

[13] R. Granek, J. Phys. II (France) 7, 1761 (1997).

[14] T. M. Bayerl, Curr. Opin. Colloid Interface Sci. 5, 232 (2000).

[15] L.C.L. Lin and F.L.H. Brown, Phys. Rev. Lett. 93, 256001 (2004).

[16] L. C. L. Lin and F. L. H. Brown, Phys. Rev. E 72, 011910 (2005).

[17] E. Evans, A. Yeung, R. Waugh, and J. Song, Springer Proc. Phys. 66, 148 (1992).

[18] G. Koster, M. VanDuijn, B. Hofs, and M. Dogterom, Proc. Natl. Acad. Sci. U.S.A. 100, 15583 (2003).

[19] E. Evans and A. Yeung, Chem. Phys. Lipids 73, 39 (1994).
[20] R. M. Raphael and R.E. Waugh, Biophys. J. 71, 1374 (1996).

[21] Y. A. Chizmadzhev, D. A. Kumenko, P. I. Kuzmin, L. V. Chernomordik, J. Zimmerberg, and F. Cohen, Biophys. J. 76, 2951 (1999).

[22] U. Seifert and S. A. Langer, Europhys. Lett. 23, 71 (1993).

[23] U. Seifert and S. A. Langer, Biophys. Chem. 49, 13 (1994).

[24] A. Yeung and E. Evans, J. Phys. II (France) 5, 1501 (1995).

[25] I. Bivas, P. Méléard, I. Mircheva, and P. Bothorel, Colloids Surf. A 157, 21 (1999).

[26] L. Miao, M. A. Lomholt, and J. Kleis, Eur. Phys. J. E 9, 143 (2002).

[27] W. Pfeiffer, S. König, J. F. Legrand, T. Bayerl, D. Richter, and E. Sackmann, Europhys. Lett. 23, 457 (1993).

[28] T. Pott and Méléard, Europhys. Lett. 59, 87 (2002).

[29] E. Lindahl and O. Edholm, Biophys. J. 79, 426 (2000).

[30] D. Chandler, Introduction to Modern Statistical Mechanics (Oxford University, New York, 1987).

[31] D. Frenkel and B. Smit, Understanding Molecular Simulation (Academic, San Diego, CA, 1996).

[32] M.P. Allen and D. J. Tildesley, Computer Simulation of Liquids (Oxford University, Oxford, 1987).

[33] R. Goetz and R. Lipowsky, J. Chem. Phys. 108, 7397 (1998).

[34] R. Goetz, G. Gompper, and R. Lipowsky, Phys. Rev. Lett. 82, 221 (1999).

[35] W. K. den Otter and W. J. Briels, J. Chem. Phys. 118, 4712 (2003).

[36] W. K. den Otter, J. Chem. Phys. 123, 214906 (2005).

[37] T. V. Tolpekina, W. K. den Otter, and W. J. Briels, J. Chem. Phys. 121, 8014 (2004).

[38] T. V. Tolpekina, W. K. den Otter, and W. J. Briels, J. Chem. Phys. 121, 12060 (2004).

[39] S. A. Shkulipa, W. K. den Otter, and W. J. Briels, Biophys. J. 89, 823 (2005).

[40] E. Freyssingeas, D. Roux, and F. Nallet, J. Phys. II (France) 7, 913 (1997).

[41] G. Althoff, D. Frezzato, G. Kothe, G. J. Moro, M. Vilfan, I. Vilfan, O. Stauch, and R. Schubert, Mol. Cryst. Liq. Cryst. 394, 93 (2003).

[42] S. J. Marrink and A. E. Mark, J. Phys. Chem. B 105, 6122 (2001). 